\title{
NONLINEAR DYNAMICS AND NON SMOOTH IN A MODEL OF SUSTAINABLE DEVELOPMENT WITH ECONOMIC EXCHANGE BETWEEN TWO SOCIETIES
}

\section{H. A. Granada ${ }^{1}$, J. A. Amador, G. Olivar}

Departamento de Ingeniería Eléctrica, Electrónica y Computación. Universidad Nacional de Colombia, Sede Manizales Campus La Nubia, Manizales, Colombia.

${ }^{1}$ hagranadadi@unal.edu.co

\begin{abstract}
Mathematical modeling in resources management has been growing in recent years, but this generally describes and studies the dynamic interaction between isolated populations and the ecological complexes to find conditions where human and natural resources coexist in a sustainable scheme. This paper will present a new proposal on two communities that are linked in a scenario of economic exchange, where trading conditions depend on the state variables and parameter values. When several communities are considered and several trading strategies are apply we get rich dynamic and nonlinear phenomena. The analysis concluded that for a sustainable scheme, some parameters must be managed inside the system in order to obtain non-trivial equilibrium or oscillatory behavior between the state variables.
\end{abstract}

Keywords: Sustainable Development, Economic Exchange, Nonlinear Dynamics.

\section{Introduction}

All previous works have studied the dynamic interaction between population and natural resources for isolated societies [1-4]. The results indicate that the success or failure of a society depends greatly on how renewable resources are managed. Specifically, the values of the parameters lead to the sustainable level of population and resources in the long term.

Up to now social-ecological systems are based on two different economic sectors: resource development and agriculture. Now, a more complex structure is introduced considering not only the two sources of income but also input and output flows of the two regions. The proposed model implies that both regions can exchange an amount of their collected renewable resources under the assumption that each region has different renewable resources ${ }^{1}$. Hereafter the subindex 1 and 2 refer to variables and parameters of regions 1 and 2 respectively. The requirement for renewable resources in the region 2 is obtained not only exploit their own resources but also an amount of resources in the region 1 . This process is analogous to region 1. This dynamic is a simple process of export-import between regions 1 and 2 .

The paper is organized as follows: In the first section a model of sustainable development 2-dimensional for one civilization is presented by [4], from this model, a 4-dimensional

\footnotetext{
${ }^{1}$ Social and environmental factors as: climate, geography and culture could determine economic activities in a region.
} 
smooth model is proposed, which involves an economic exchange between two civilizations (2), this model can be viewed as a non smooth model if the parameters of this system change abruptly over time. The second section is aimed to obtain results of the smooth model where behaviors with and without economical interchange are highlit, later continuations of equilibrium points and Hopf points are made which allow to understand the change of stability of the equilibrium points. In the third section the non smooth system is introduced and shows some interesting behavior of this system. In the fourth section the conclusions of this work are given, the fifth section future researches are proposed. Finally the theoretical framework used in this work.

\section{Mathematical model}

The interaction between population and renewable resources can be described by Ricardo-Malthus model proposed in $[1,4]$. This model assumes that the dynamics of resources depends on the natural growth and the rate of collection of these. On the other hand, the population dynamics depends on the difference between calories consumed and the per capita level of calories needed to survive as presented in the following system of differential equations (ODEs)

$$
\left\{\begin{array}{l}
\dot{S}=\left[\rho\left(\frac{S}{k}-1\right)\left(1-\frac{S}{K}\right)-\alpha \beta L\right] S \\
\dot{L}=\gamma\left(\lambda(1-\beta)^{\delta} L^{\delta-1}+\bar{\phi} \alpha \beta S-\bar{\sigma}\right) L
\end{array}\right.
$$

Let us suppose that the population in both regions produce the same agricultural product, ie corn, but collect different renewable resources, for example the region 1 exploiting the land to produce wood and the region 2 using the sea for fishing. In this sense the dynamics of each region can be modeled by a ordinary differential equations system (1) but with different values of parameters (see Table 1). Now, let us suppose $C_{1}, C_{2} \in[0,1]$ are portions of resources exploited by regions 1 and 2 and used for their own consumption respectively, where $\left(1-C_{2}\right)$ and $\left(1-C_{1}\right)$ are parts of resources that the region 1 and region 2 receive one of each respectively.

Table 1. Parameter values

\begin{tabular}{cl|cl}
\hline Parameter & Value & Parameter & Value \\
\hline$\beta_{1}$ & 0.3 & $\beta_{2}$ & 0.4 \\
$\alpha_{1}$ & 0.0001 & $\alpha_{2}$ & 0.00011 \\
$\rho_{1}$ & 0.025 & $\rho_{2}$ & 0.04 \\
$\lambda_{1}$ & 12.95 & $\lambda_{2}$ & 12.95 \\
$\delta_{1}$ & 0.7 & $\delta_{2}$ & 0.7 \\
$k_{1}$ & 700 & $k_{2}$ & 600 \\
$K_{1}$ & 12000 & $K_{2}$ & 14000 \\
$\gamma_{1}$ & 0.1 & $\gamma_{2}$ & 0.1 \\
$\sigma_{1}$ & 0.14 & $\sigma_{2}$ & 0.14 \\
$\phi_{1}$ & 0.3 & $\phi_{2}$ & 0.25 \\
\hline
\end{tabular}

The import process is summarized by the consumption of calories that each region receives from the other, then the region 1 consumes $C_{1} \phi_{1} \alpha_{1} \beta_{1} S_{1} L_{1}$ caloric units of their 
own resources and exports $\left(1-C_{1}\right) \phi_{1} \alpha_{1} \beta_{1} S_{1} L_{2}$ to region 2 . The same process occurs in the other direction, since the region 2 consumes $C_{2} \phi_{2} \alpha_{2} \beta_{2} S_{2} L_{2}$ and exports to the region 1 $\left(1-C_{2}\right) \phi_{2} \alpha_{2} \beta_{2} S_{2} L_{1}$. On the other hand, assuming that each region avoids harvesting an amount of resources with caloric value equal to the caloric value that they have imported, thus:

$$
\begin{aligned}
S_{2-1} & =\frac{\left(1-C_{2}\right) \phi_{2} \alpha_{2} \beta_{2} S_{2}}{\phi_{1} \alpha_{1} \beta_{1}} \\
S_{1-2} & =\frac{\left(1-C_{1}\right) \phi_{1} \alpha_{1} \beta_{1} S_{1}}{\phi_{2} \alpha_{2} \beta_{2}}
\end{aligned}
$$

where $S_{i-j}$ is the amount of resources in the region $j$ which has a caloric value equivalent to resources imported from the region $i$.

$$
\left\{\begin{array}{l}
\dot{S}_{1}=\rho_{1}\left(\frac{S_{1}+S_{2-1}}{k_{1}}-1\right)\left(1-\frac{S_{1}+S_{2-1}}{K_{1}}\right) S_{1}-\alpha_{1} \beta_{1} L_{1}\left(S_{1}-S_{2-1}\right) \\
\dot{L}_{1}=\left(\gamma_{1} \lambda_{1}\left(1-\beta_{1}\right)^{\delta_{1}} L_{1}^{\delta_{1}-1}+C_{1} \phi_{1} \alpha_{1} \beta_{1} S_{1}+\left(1-C_{2}\right) \phi_{2} \alpha_{2} \beta_{2} S_{2}-\sigma_{1}\right) L_{1} \\
\dot{S}_{2}=\rho_{2}\left(\frac{S_{2}+S_{1-2}}{k_{2}}-1\right)\left(1-\frac{S_{2}+S_{1-2}}{K_{2}}\right) S_{2}-\alpha_{2} \beta_{2} L_{2}\left(S_{2}-S_{1-2}\right) \\
\dot{L}_{2}=\left(\gamma_{2} \lambda_{2}\left(1-\beta_{2}\right)^{\delta_{2}} L_{2}^{\delta_{2}-1}+C_{2} \phi_{2} \alpha_{2} \beta_{2} S_{2}+\left(1-C_{1}\right) \phi_{1} \alpha_{1} \beta_{1} S_{1}-\sigma_{2}\right) L_{2}
\end{array}\right.
$$

The system (2) is a smooth model if we assume that the economic exchange constant $C_{i}$ are fixed. Now if they vary abruptly over time, make the system to become a discontinuous non smooth system. In the following sections each one of these systems will be studied in detail.

\section{Smooth system results}

The system (2) is a set of ordinary differential equations coupled to depend not only on the state variables and parameter values, but also the exchange rates $C_{1}$ and $C_{2}$. Three scenarios may occur: Non economic exchange, $C_{i}=1$; economic exchange, $C_{i} \neq 1$, export of the region $i$ to the region $j, C_{i}=1, C_{j} \neq 1$ if $i \neq j$.

As it follows we assume that both civilizations have large amount of resources and low population in a time $t_{0}$, that is why the initial conditions are fixed for each civilization like the values of the parameters given by Table 1 . It is emphasized that only the analysis of sensitivity to economic exchange parameters is made in this work.

Below several scenarios are presented, where sensitivity of the system can be appreciated respect to the parameter values of economic exchange $C_{i}$.

\subsection{Without economic exchange}

The Figure 1 shows that taking similar initial conditions for both civilizations, the temporal evolution of 1(a) reaches a sustainable development, while 1(b) runs out its resources, 
this is because in civilization 2 the rate of resource exploitation is greater than in civilization 1 and a large percentage of the population is dedicated to exploit this resource leading to exhaustion.

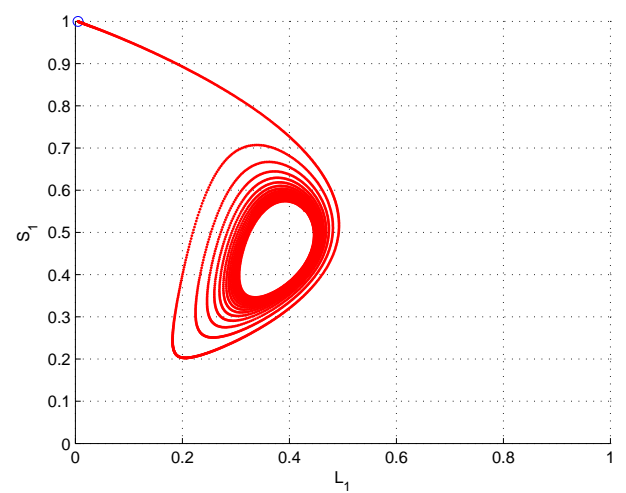

(a) Civilization 1

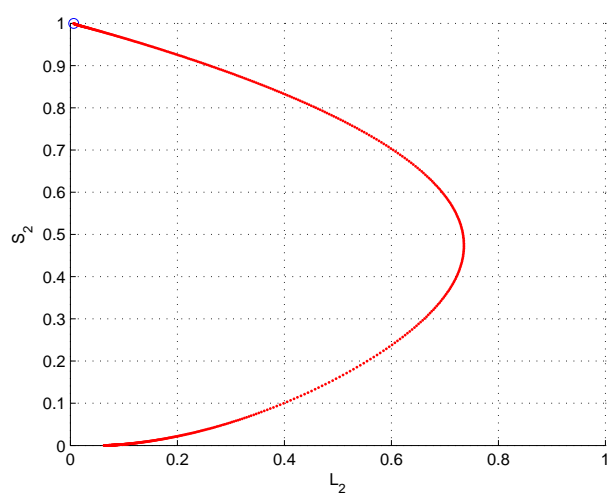

(b) Civilization 2

Figure 1. $C_{1}=1$ and $C_{2}=1$

\subsection{Exporting the region $i$ to region $j$}

If civilization 2 of Figure 1(b) had an external supply of resources that could help preserve their own resources allowing this way to keep a sustainability. As an example, let us assume that a civilization 1 supplies the $10 \%$ of all the products collected to civilization 2 , then this will help both populations are sustainable as shown below.

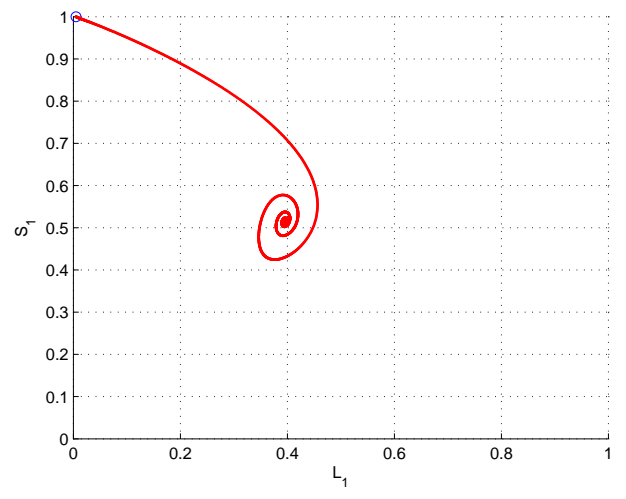

(a) Civilization 1

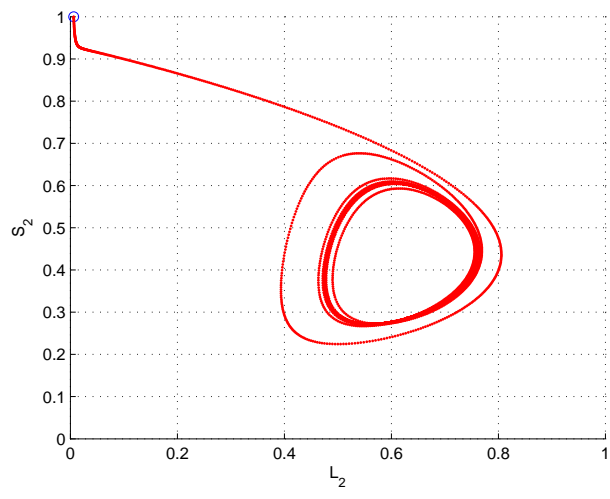

(b) Civilization 2

Figure 2. $C_{1}=0.9$ and $C_{2}=1$

Figure 2 shows that 2(a) reaches the $45 \%$ of the maximum capacity of population, since it is affected by sending resources to another population without receiving any benefits, compared with $50 \%$ of the maximum capacity of population as shown in 1(a) where there is no economic exchange. Moreover, the constant supply of civilization 1 to civilization 2 allowed in 2(b) an increase in the population up to $80 \%$ of the maximum capacity of population. 


\subsection{Economic exchange}

If civilization 2 has not reached the sustainable development due to lack of food sources and the regeneration rate of renewable resource, it is necessary to have an economic exchange between two populations with different resources so as to obtain sustainability between the two regions. This is accomplished for example by requiring the two populations with an exchange of $10 \%$ towards the other population of what is produced as shown in Figure 3.

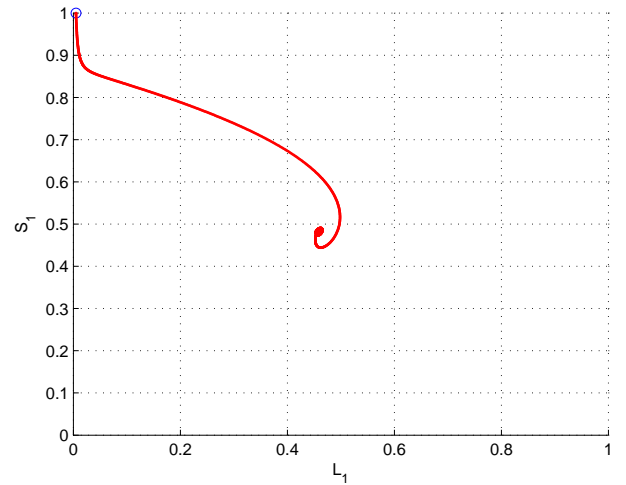

(a) Civilization 1

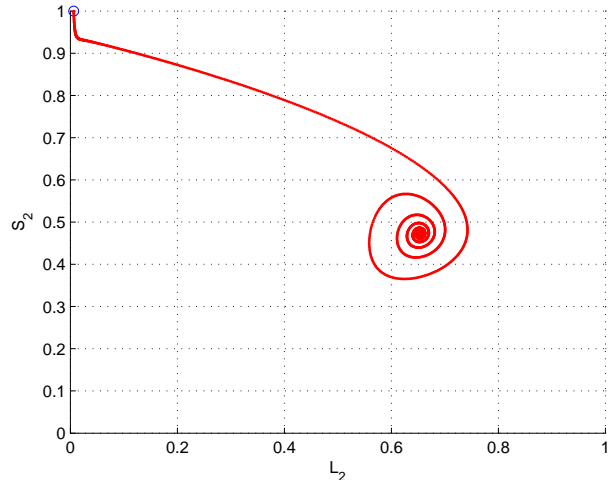

(b) Civilization 2

Figure 3. $C_{1}=0.9$ and $C_{2}=0.9$

Civilization 3(a) reaches a maximum of $50 \%$ of the maximum capacity of the population and resources are stabilized near the $50 \%$ of the maximum capacity of resources, while civilization 3(b) reaches a maximum of $75 \%$ of the maximum capacity of the population and about $50 \%$ of the resource capacity at steady state. This dynamic interaction of trade allows the two regions benefit and achieve sustainable development.

\subsection{Steady state analysis}

As seen in previous results, the parameters $C_{i}$ with $i \in\{1,2\}$ are of great importance in determining the sustainability of civilizations. It is for this reason that this section is dedicated to an analysis of the stationary state of the system (2).

To do this, we turn to smooth systems theory see [5]. The implementation of the algorithms of the simulations in this section were performed using the numerical methods exposed in [6].

Our intention now is to determine how can the sustainability of both civilizations be interpreted. In the case of a civilization, sustainable development is achieved when starting from fixed initial condition, the trajectory converges to a stable equilibrium point whose state variables are not null, because if the state variable resource $S=0$, this implies that the population has exhausted its renewable resource and therefore it is necessary to the exploitation of other renewable resource.

Another scenario for the sustainability is that the system starting from a fixed condition would converge to a stable limit cycle, this is why it is necessary to calculate the Hopf bifurcation to ensure from that point you get these stable limit cycles. 


\subsubsection{Continuation of equilibrium points}

To ensure the sustainability of both regions is necessary to make an uniparametrical analysis of $C_{2}$ leaving the parameter $C_{1}=0.9$ fixed, to do this we calculate the value of the parameter $C_{2}$ for which we have a Hopf bifurcation and the stability of equilibrium points System (2) is classified by using a numerical continuation of equilibria, as seen in Figure 4.

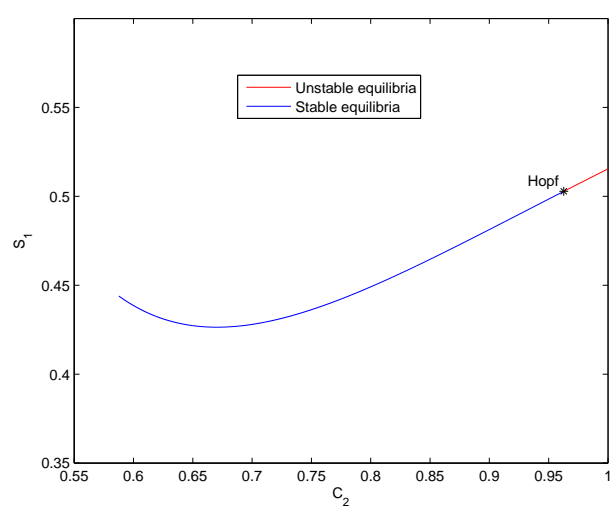

(a) Equilibria continuation

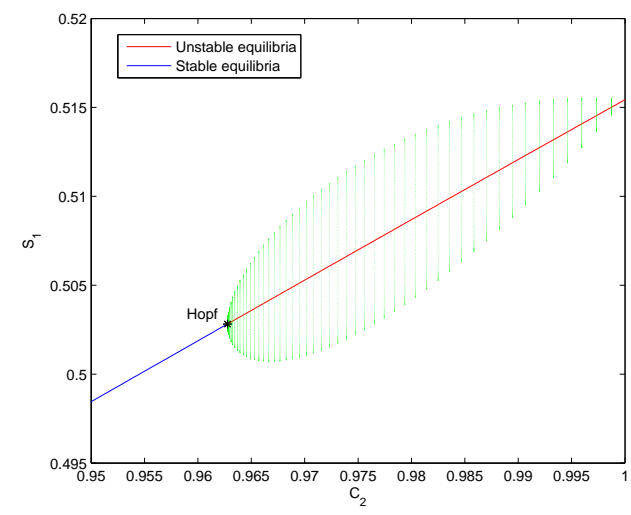

(b) Family of limit cycles

Figure 4. Equilibria continuation

In Figure 4(a) shows how it affects the exchange parameter $C_{2}$ to renewable resources of civilization 1 in steady state. The blue curve contains points of stable equilibrium, after Hopf bifurcation $\left(C_{2}=0.9627\right)$ these points become unstable equilibrium points giving origin to stable limit cycles.

On the other hand, depending on the initial conditions of the system and a fixed value of $C_{2}$, the evolution of the system may or may not converge to a point on this curve which could be determined by calculating the attraction zones, i.e the set of initial conditions that converge to the same equilibrium point.

Figure 4(b) shows the family of limit cycles that arise from the Hopf bifurcation.

\subsubsection{Continuation of Hopf points}

As shown in previous simulations, Hopf point determines the change of system stability by setting the parameter $C_{1}=0.9$, now there will be a two-parameter continuation of the system (2), where parameters to vary are the $C_{i}$ of economic exchange, as shown in Figure 5 , which shows the curve of Hopf points as we vary the parameters $C_{i}$, this curve can divide two regions, which indicate when the equilibrium points go from being stable to unstable originating a family of limit cycles.

\section{Non smooth System Results}

Economic exchange occurs not only constant, it can also be in the implementation of policies for resource interchange designed for each civilization. These policies for exchange are defined in terms of population and resources. To do this, we define two regions of the 


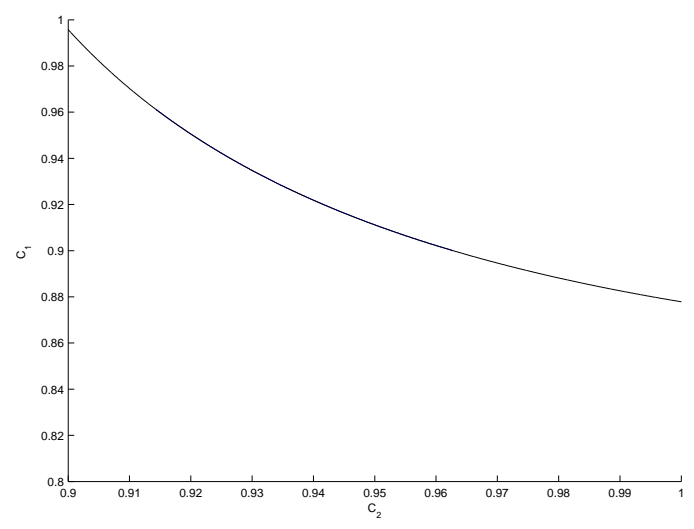

Figure 5. Hopf curve

exchange in each civilization $i \in\{1,2\}$ and $R_{i}=\left[L_{\text {min }}^{i}, L_{\text {max }}^{i}\right] \times\left[S_{\text {min }}^{i}, S_{\text {max }}^{i}\right]$. Defining these areas of economic exchange make the system (2) becomes a non-smooth system appearing abrupt changes in the evolution of its state variables. Such systems are called discontinuous or piecewise smooth system, also known under the name Filippov systems [7]:

$$
\dot{x}=f(x(t)), \quad x \in \mathbb{R}^{n}
$$

where $f$ is discontinuous at the border of $R_{i}$.

In defining the areas of economic exchange, four vector fields are defined which make the system (3) to be discontinuous, for these systems we use different methods of bifurcacional analysis to those used in smooth systems. In this paper we do not consider the Filippov system analysis, only limit ourselves to show how the control regions of economic exchange influence in the sustainable development of both civilizations.

In the following simulations we will adopt three basic colors as a notation indicating the three possible scenarios of exchange:

- Red: No economic exchange $C_{i}=1$.

- Blue: Economic exchange between the two civilizations $C_{i}=0.9$.

- Green: Economic exchange in one direction $C_{i}=1$ and $C_{j}=0.9$ if $i \neq j$.

\subsection{Economic exchange: Causes unsustainibility in civilizations}

The poor policy decisions about economic exchange can carry devastating problems for civilizations, because they affect directly to renewable resources and each population. To see this, we define the exchange regions as shown in Figure 6.

When civilization 1 was reaching its maximum of population there was an economic exchange where civilization 2 sent their corresponding resources in a short time, which meant that resources of population 1 were exhausting, and then two civilizations collapsed, phenomenon can be explained by poor management of renewable resources. 


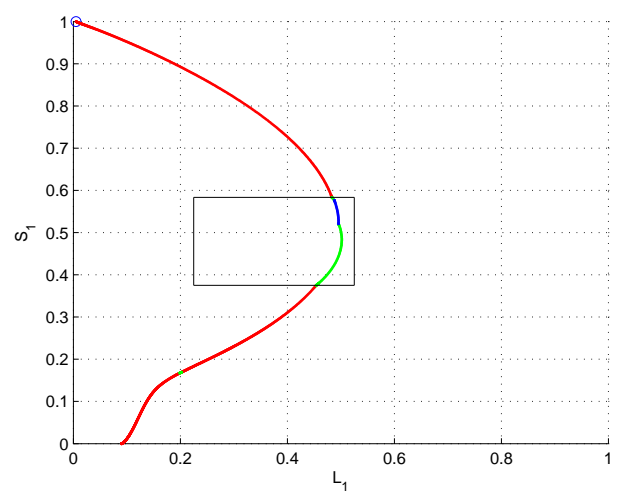

(a) Civilization 1

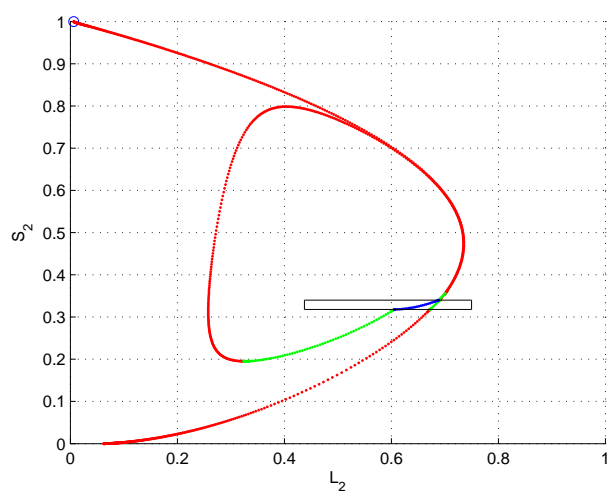

(b) Civilization 2

Figure 6. Exchange: Unsustainable - Unsustainable

\subsection{Economic exchange: Causes Sustainability}

A good selection of exchange regions allow to obtain two possible scenarios, that given time enough a region sustains another without receiving anything in return or that the two civilizations always keep an economic exchange allowing them to benefit mutually.

\subsubsection{Unilateral exchange: Causes sustainability}

In Figures 2(b) and 3(b) we see that the financial assistance of 1 is of vital importance to the sustainability of 2 . Now, if this civilization would have been more generous in making exchanges could have prevented the collapse of civilization 1 and then get permanently the help of this civilization reaching sustainable development for both, as shown in the following simulation (Figure 7).

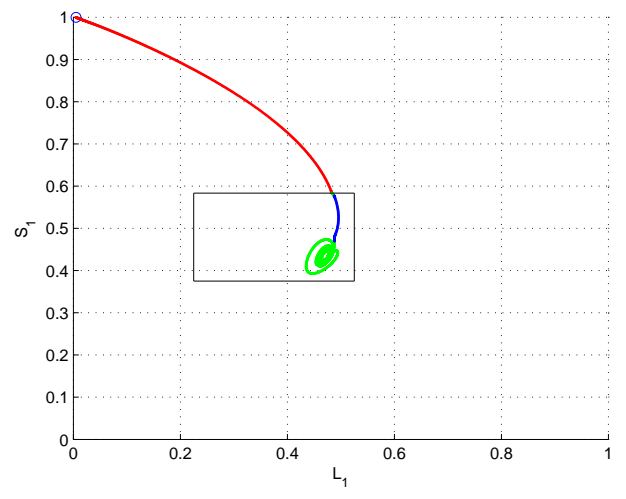

(a) Civilization 1

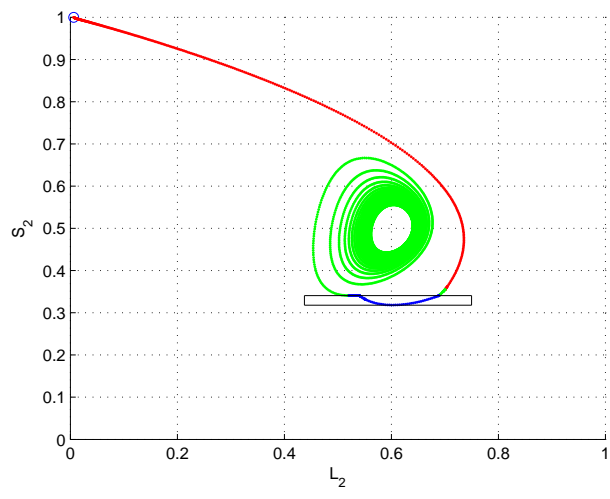

(b) Civilization 2

Figure 7. Unilateral exchange: Sustainable - Sustainable

Figure 7 shows that with little financial help of 2 can make the system to be sustainable because it has a unilateral exchange at steady state by 1 to 2 . 


\subsubsection{Bilateral exchange: Causes Sustainability}

Now if policies of economic exchange would be implemented in appropriate regions, a sustainable development could be held for both civilizations in which in steady-state case a bilateral or mutual contribution can be found, Figure 8. Exchange at steady state by 1 to 2 .

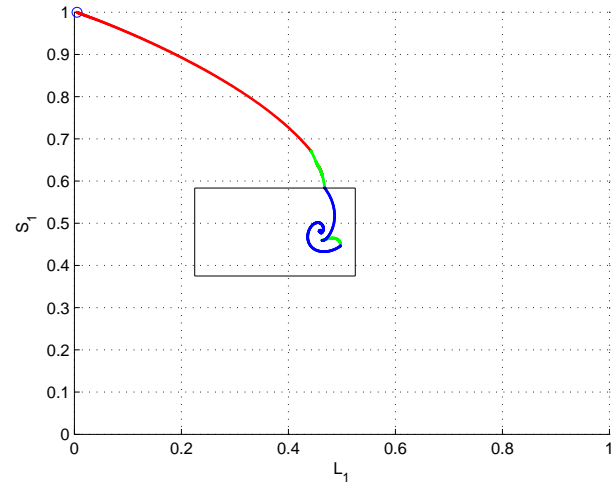

(a) Civilization 1

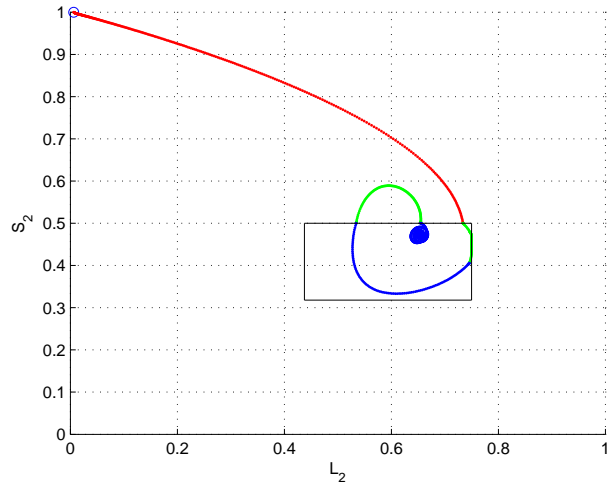

(b) Civilization 2

Figure 8. Bilateral exchange: Sustainable - Sustainable

\subsection{Exchange: Causes unsustainability in a civilization}

Returning to Figure 6(a) and defining another exchange zone to civilization 1, the scenario is different because this civilization reaches its sustainable development while civilization 2 runs out its renewable resource as shown in the simulation of Figure 9.

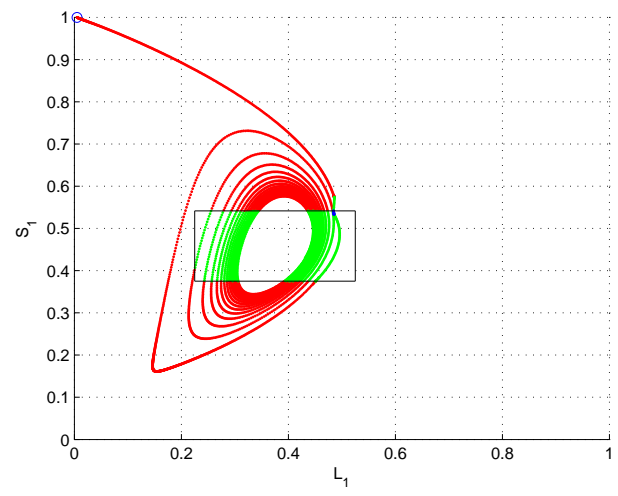

(a) Civilization 1

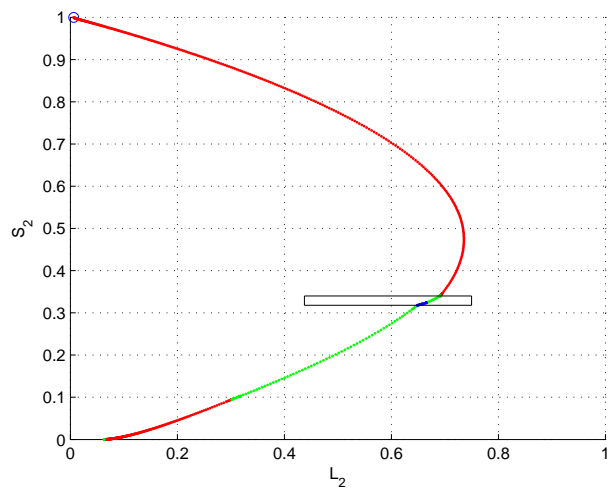

(b) Civilization 2

Figure 9. Exchange: Sustainable - Unsustainable

\section{Conclusions}

1 The model proposed in [4] allows a study of how the economy of community can be affected when there is economic exchange. 
2 Economic exchange between communities contributes to development as to the sustainability of several communities.

3 A bifurcacional analysis of the system (2) can determine the ranges of parameter values for which the civilizations reach their sustainable development.

4 Exchange zones permit the design and implementation of control actions on the economy of each sector.

5 The stability of the equilibrium points as the Hopf bifurcation detection is key to sustainable development in a region.

\section{Future work and research approaches}

1 The model can be complemented by involving CES production functions, migration models, demographic, macroeconomic theory and climate change.

2 The dynamic interaction of economic exchange among $n$ regions can be seen as a neural network giving origin to a wide path of sustainable development research.

3 The characterization of other local bifurcations (Hopf, Fold) as global ones (Homoclinic) allow to mark somes of $m$-parametric sustainability.

4 A detailed bifurcational study over the most relevant patterns of the system with interchange by using Filippov's theory.

\section{Acknowledgements}

We would like to thank the research Groups ABC Dynamics and PCI of "Universidad Nacional of Colombia" Manizales, for their support on this work and to "Centro de Excelencia en Investigación Básica y Aplicada" (CEIBA) for their financial support. 


\section{References}

[1] Brander J. A., Taylor M. S., "The Simple Economics of Easter Island: A Ricardo-Malthus Model of Renewable Resource Use". The American Economics Review. 88, 119-138, 1998.

[2] Dalton T. R., Coast R. M., “Could institutional reform have saved Easter Island?”. Journal of Evolutionary Economics. 10, 489-505, 2000.

[3] Reuveny R., Decker C. S., "Easter Island: historical anecdote or warning for the future?". Ecological Economics. 35, 271-271, 2000.

[4] D'Alessandro S., "Non-linear dynamics of population and natural resources: The emergence of different patterns of development". Ecological Economics. 62, 473-481, 2007.

[5] Kuznetsov, Yuri A., "Elements of Applied Bifurcation Theory". Springer-Verlang New York., 2004.

[6] Govaerts, Willy J. F., "Numerical Methods for Bifurcations of Dynamical Equilibria". SIAM-Philadelphia., 2000.

[7] Filippov A.F., "Differential equations with discontinuous right-hand sides". American Mathematical Society Translations. Series 2, AMS, Ann Arbor 199-231, 1964. 